Int. J.Morphol.,

25(4):885-894, 2007.

\title{
Formaciones Venosas de la Fosa Cubital en el Individuo Mapuche. Estudio Bioscópico
}

\author{
Venous Formations in the Cubital Fossa of Mapuche. Bioscopy Study \\ Mariano del Sol; Miguel Lagos Mardones \& Edison Torres Bustos
}

\begin{abstract}
DEL SOL, M.; LAGOS, M. M. \& TORRES, B. E. Formaciones venosas de la fosa cubital en el individuo mapuche. Estudio bioscópico. Int. J. Morphol., 25(4):885-894, 2007.

RESUMEN: Las venas superficiales de la fosa cubital constituyen uno de los sitios más importantes para punción venosa. La disposición de esas venas presenta numerosas variaciones y, la disposición de ellas no ha sido estudiada en el grupo étnico mapuche. Debido a esto, y considerando a este grupo como el mayor conglomerado étnico de América del Sur, efectuamos este estudio. Fueron analizadas las venas superficiales de la fosa cubital, en 300 miembros superiores ( 150 derechos y 150 izquierdos) de individuos de ambos sexos (30 hombres y 120 mujeres), chilenos del grupo étnico mapuche, con edades entre 15 y 84 años. El estudio fue realizado en las postas rurales adyacentes a la ciudad de Temuco. Basados en la clasificación de del Sol et al. (1988) para las formaciones venosas de la fosa cubital, obtuvimos los siguientes resultados: Tipo I $(38,7 \%)$, la vena cefálica (VC) se divide en vena intermedia basílica (VIB) y vena intermedia cefálica (VIC), uniéndose a la vena basílica (VB) y vena cefálica accesoria (VCA), respectivamente. La VIB era de mayor calibre y la vena intermedia del antebrazo (VIA) drenaba, generalmente, en la VB; Tipo II (28,3\%), la VC origina la vena intermedia del codo (VICo), que se une a la VB. No existe VCA, siendo la VICo de mayor calibre y la VIA drena en la VB; Tipo III (24\%), no existe comunicación entre la VB y VC a nivel de la fosa cubital. La VIA drena en la VB; Tipo IV (4,3\%), la VC drena en la VB y la VIA drena en la VC; Tipo V otras disposiciones, donde se incluye la M clásica (1\%) que resulta de la división de la VIA. La utilización de las VIC y VC se recomienda cuando ellas tienen un calibre semejante a la VIB o a la VICo ya que los riesgos de punción de otras estructuras anatómicas importantes, como ramos anteriores del nervio cutáneo medial del antebrazo o arteria braquial, son mínimos.
\end{abstract}

PALABRAS CLAVE: Anatomía; Venas de la fosa cubital; Sitios de punción venosa.

\section{INTRODUCCIÓN}

Es importante conocer la distribución de las venas superficiales de la fosa cubital en el Hombre y sus relaciones con las diversas estructuras anatómicas de la región. Estas venas son utilizadas, preferencialmente, como sitios de punción, en la recolección de muestras para su estudio en el laboratorio, y en las últimas décadas, están siendo usadas para la introducción de catéteres en la obtención de sangre desde las cámaras cardiacas y para la realización de angiocardiografías, entre otros procedimientos.

Estas venas, tienen estrecha relación con el sistema arterial, principalmente con la arteria braquial y con las eventuales variaciones anatómicas ocurridas en la fosa cubital, como la presencia de las arterias radial y ulnar superficiales.

Así, una inyección intra o para-arterial accidental puede ocasionar desde un espasmo hasta una completa obstruc- ción, con secuelas tan importantes como la amputación del segmento afectado (Cagnon, 1966; Last, 1968).

En la literatura médica mundial, existen numerosas publicaciones sobre la distribución venosa superficial en la fosa cubital. Estos estudios se han realizado en diversos grupos étnicos, incluyendo trabajos en individuos chilenos de ascendencia hispánica (Skewes, 1944) y en jóvenes chilenos de diferentes grupos étnicos (del Sol et al., 1990); sin embargo, no existen publicaciones sobre las formaciones venosas en la fosa cubital en el grupo étnico mapuche.

Escasos son los trabajos sobre Antropología Biológica y Física de los individuos del grupo étnico mapuche, destacándose algunos trabajos realizados por Guevara (1927); Henkel (1958) y recientemente, por del Sol \& Hunter (2004) y del Sol $(2005,2006)$. 
El conocimiento de la distribución venosa superficial de la fosa cubital en el grupo étnico mapuche, es importante, no sólo desde los puntos de vista anátomo-clínico y quirúrgico, sino también, antropológico físico y biológico, considerando que constituye el mayor conglomerado étnico de América del Sur, distribuyéndose ampliamente en el Sur de Chile y, principalmente, en la IX Región de Chile.

\section{MATERIAL Y MÉTODO}

Fueron analizadas las venas superficiales de la fosa cubital en 300 miembros superiores (150 derechos y 150 izquierdos) de individuos de ambos sexos (30 hombres y 120 mujeres), chilenos, del grupo étnico mapuche, con edades entre 15 y 84 años. El estudio se realizó en 14 postas rurales y consultorios próximos a la ciudad de Temuco, en la provincia de Cautín de la IX Región de Chile. Contando con el consentimiento informado, se utilizó una ficha de identificación para cada individuo, indentificándolos con sus nombres y apellidos y los y de sus padres, lugar de nacimiento, edad y sexo. Se elaboró a escala un esquema de los miembros superiores, donde se esquematizó la distribución venosa de la regiones antebraquiales anterior y cubital.

Con el fin de visualizar mejor las venas de la región cubital, se colocó un torniquete en la parte inferior del tercio medio del brazo. En caso que las venas aún no fuesen visibles, fue aplicado calor regional, a través de un masaje en la región en estudio. Fueron descartados los individuos donde no fue posible visualizar con claridad, las venas de la región antebraquial anterior, garantizando la validez de las observaciones.

\section{TIPO I}

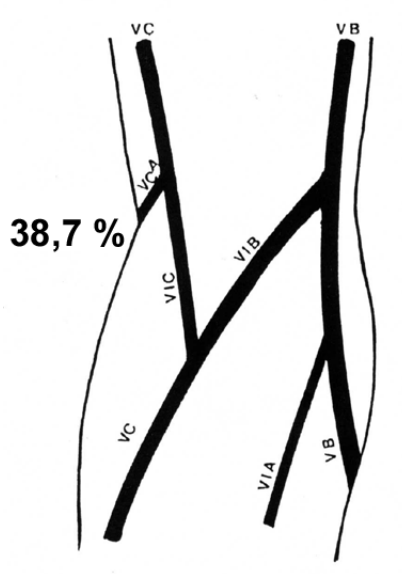

TIPO II

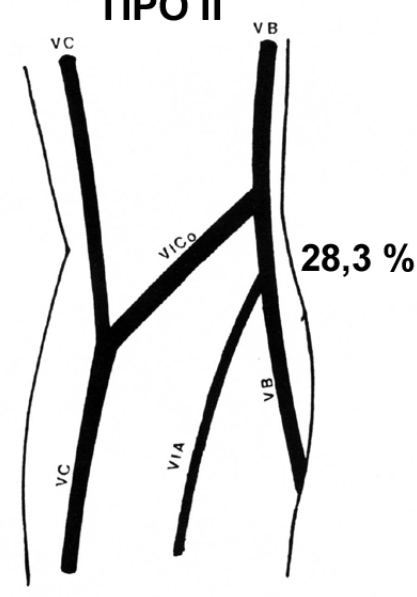

TIPO III

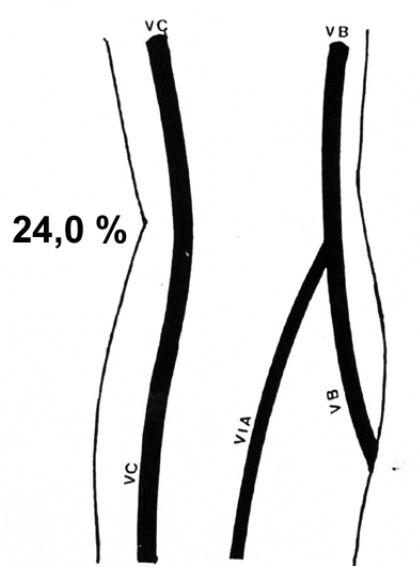

TIPO IV

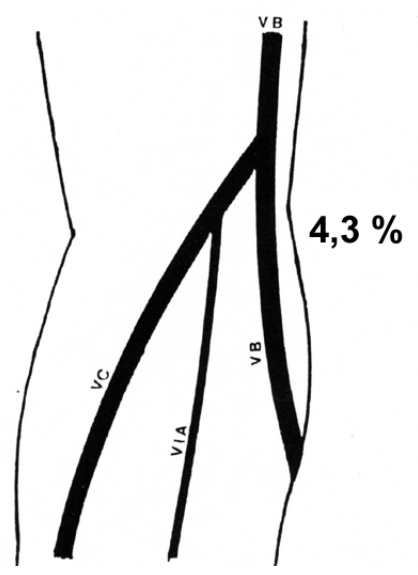

Fig. 1. Formaciones venosas Tipos I, II, III y IV en la fosa cubital de 150 individuos de ambos sexos del grupo étnico mapuche.

Los patrones de drenaje venoso en la fosa cubital fueron clasificados de acuerdo a la tipología propuesta por del Sol et al. (1988), en recién nacidos brasileños.

Tipo I (38,7\%), la vena cefálica (VC) se divide en vena intermedia basílica (VIB) y vena intermedia cefálica (VIC), uniéndose a la vena basílica (VB) y vena cefálica accesoria (VCA), respectivamente. La VIB era de mayor calibre y la vena intermedia del antebrazo (VIA) drenaba generalmente en la VB; Tipo II (28,3\%), la VC origina la vena intermedia del codo (VICo), que se une a la VB. No existe VCA, siendo la VICo de mayor calibre y la VIA drena en la VB; Tipo III (24\%), no existe comunicación entre la VB y VC a nivel de la fosa cubital. La VIA drena en la VB; Tipo IV (4,3\%), la VC drena en la VB y la VIA drena en la VC; Tipo $\mathrm{V}$ otras disposiciones, donde se incluye la M clásica $(1 \%)$ que resulta de la división de la VIA.

\section{RESULTADOS}

Debido fundamentalmente a la gran sinonimia de los términos que serán abordados, se hizo necesaria la utilización de los términos que aparecen en la Nomina Anatomica aprobada por el IANC, el año 1985 en Londres y publicada en Edimburgo en 1989. No se utilizó la Terminología Anatómica actual porque dejó de lado las VIC y VIB, elementos fundamentales de la división de la $\mathrm{VC}$ del antebrazo a nivel de la fosa cubital. 
DEL SOL, M.; LAGOS, M. M. \& TORRES, B. E. Formaciones venosas de la fosa cubital en el individuo mapuche. Estudio bioscópico. Int. J. Morphol., 25(4):885-894, 2007.

Tabla I. Formaciones venosas en la fosa cubital, de ambos lados, en 150 individuos de ambos sexos, del grupo étnico mapuche.

\begin{tabular}{|c|c|c|c|c|c|c|}
\hline \multirow[b]{2}{*}{ Tipos } & \multicolumn{2}{|c|}{ Lado derecho } & \multicolumn{2}{|c|}{ Lado izquier do } & \multicolumn{2}{|c|}{ Total } \\
\hline & $\mathrm{n}$ & $\%$ & $\mathrm{n}$ & $\%$ & $\mathrm{n}$ & $\%$ \\
\hline I & 63 & 42,0 & 53 & 35,3 & 116 & 38,7 \\
\hline II & 47 & 31,4 & 38 & 24,3 & 85 & 28,3 \\
\hline III & 29 & 19,3 & 43 & 28,8 & 72 & 24,0 \\
\hline IV & 5 & 3,3 & 8 & 5,3 & 13 & 4,3 \\
\hline V & 6 & 4,0 & 8 & 5,3 & 14 & 4,7 \\
\hline Total & 150 & 100,0 & 150 & 100,0 & 300 & 100,0 \\
\hline
\end{tabular}
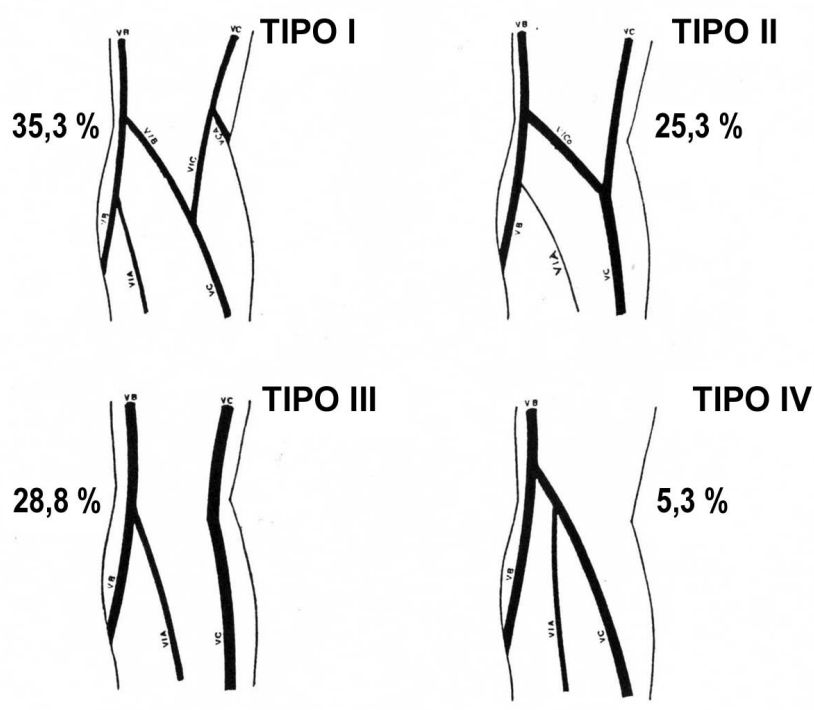

FOSA CUBITAL IZQUIERDA
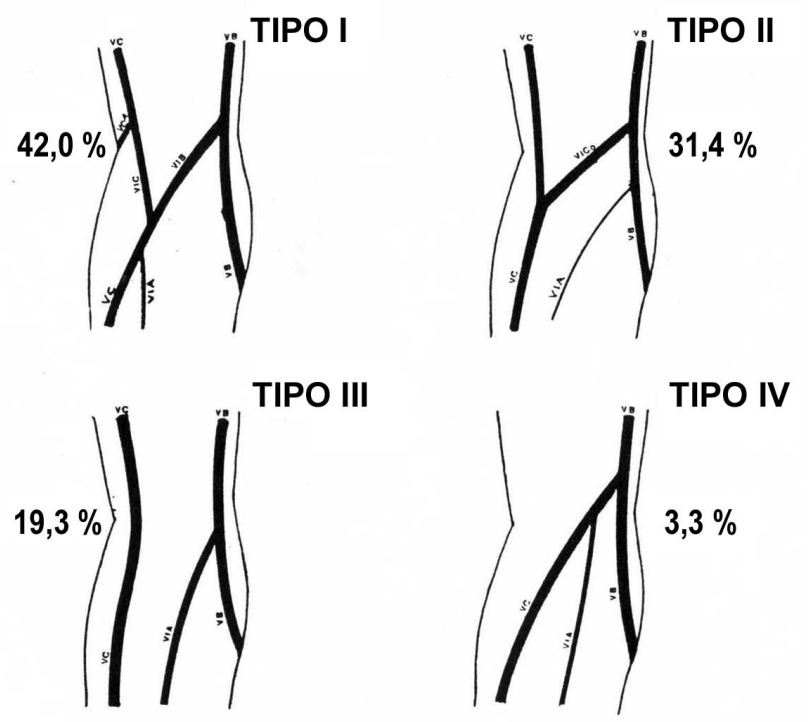

FOSA CUBITAL DERECHA

Fig. 2. Formaciones venosas Tipos I, II, III y IV en 150 fosas cubitales izquierdas y 150 derechas, de individuos de ambos sexos del grupo étnico mapuche.

Los diferentes tipos de formaciones venosas en la fosa cubital de individuos mapuches de ambos sexos y en ambos lados, las podemos observar en la Tabla I y Fig. 1, su distribución de acuerdo al lado en ambos sexos en las Fig. 2. La distribución de estas venas por lado, sexo y tipo de formación se encuentra expresado en la Figs. $3 a$ y $3 b$.

El calibre comparativo de las venas de la fosa cubital, en individuos de ambos sexos, con los tipos de formaciones venosas I y II se encuentra en las Tablas II y III.

La M clásica, formada por la división de la VIA en VIB y VIC, a nivel de la fosa cubital, se presentó en sólo 3 casos (dos miembros superiores izquierdos y uno derecho) en el sexo femenino. Estos 3 casos constituyen el 1\% de la muestra y fueron incluidos en el tipo $\mathrm{V}$ de nuestro estudio.

La frecuencia de la VIA en las formaciones venosas Tipos I, II y III de la fosa cubital de individuos mapuche, de ambos sexos, se presenta en la Tabla IV. En 6 casos en el Tipo I se observaron dos VIA y en un caso en el Tipo II.

El drenaje de la VIA en las formaciones venosas Tipos I, II y III de la fosa cubital de individuos mapuche, de ambos sexos, se presenta en la Tabla V.

De los 13 casos de Tipo IV la VIA se encontró en 8 $(61,5 \%)$, drenando, en individuos de ambos sexos, en todos los casos en la VC. 

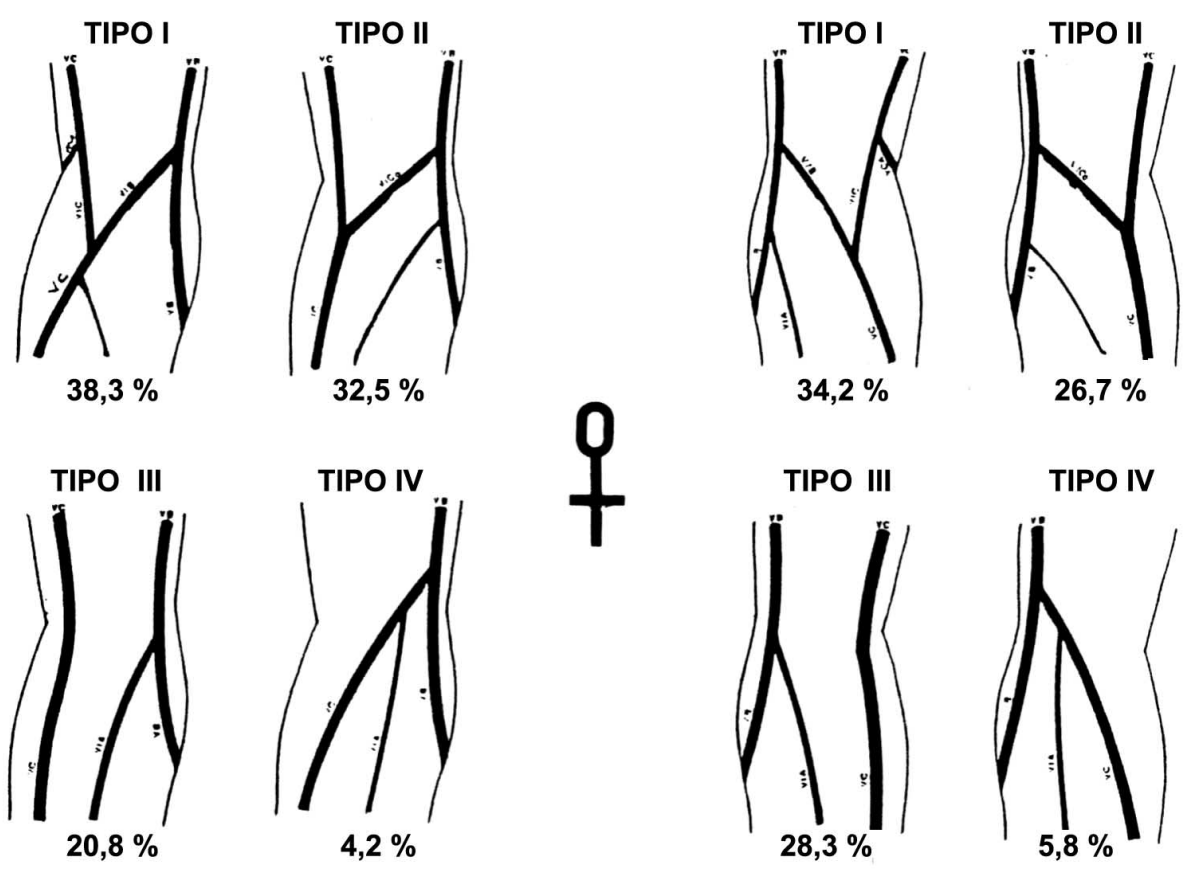

FOSA CUBITAL DERECHA

FOSA CUBITAL IZQUIERDA

Fig. 3a. Formaciones venosas Tipos I, II, III y IV en fosas cubitales derecha e izquierda, de individuos de sexo femenino del grupo étnico mapuche.

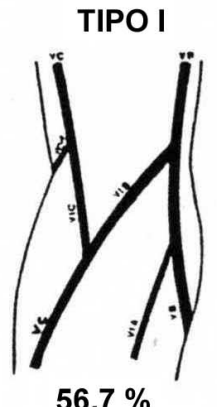

$56,7 \%$

TIPO III

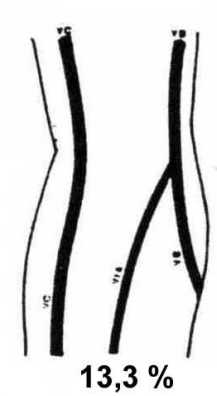

$13,3 \%$

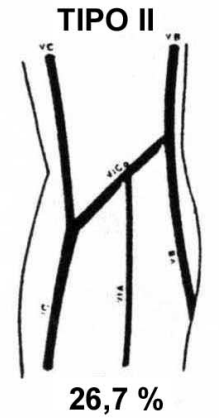

TIPO IV

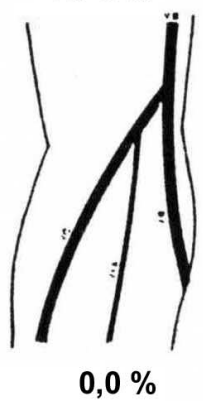

FOSA CUBITAL DERECHA

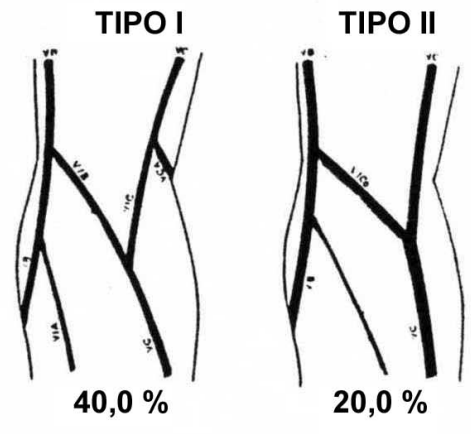

TIPO III

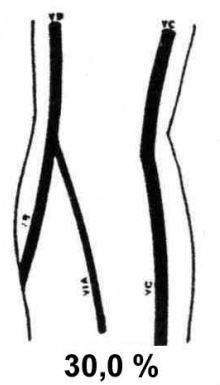

TIPO IV

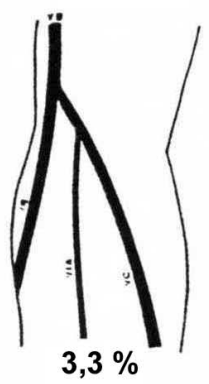

FOSA CUBITAL IZQUIERDA

Fig. 3b. Formaciones venosas Tipos I, II, III y IV en fosas cubitales derecha e izquierda, de individuos de sexo masculino del grupo étnico mapuche. 
Tabla II. Calibre mayor de las venas superficiales de la fosa cubital, derecha e izquierda, en individuos de ambos sexos, del grupo étnico mapuche, con formaciones venosas Tipo I.

\begin{tabular}{|c|c|c|c|c|c|c|}
\hline & \multicolumn{2}{|c|}{ Lado derecho } & \multicolumn{2}{|c|}{ Lado izquier do } & \multicolumn{2}{|c|}{ Total } \\
\hline Venas & $\mathrm{n}$ & $\%$ & $\mathrm{n}$ & $\%$ & $\mathrm{n}$ & $\%$ \\
\hline VIB & 26 & 41,3 & 22 & 41,5 & 48 & 41,4 \\
\hline VIC & 20 & 31,7 & 13 & 24,6 & 33 & 28,4 \\
\hline $\mathrm{VC}$ & 2 & 3,2 & 4 & 7,5 & 6 & 5,2 \\
\hline VB & 5 & 7,9 & 4 & 7,5 & 9 & 7,8 \\
\hline $\mathrm{VIB}=\mathrm{VIC}$ & 10 & 15,9 & 10 & 18,9 & 20 & 17,2 \\
\hline Total & 63 & 100,0 & 53 & 100,0 & 116 & 100,0 \\
\hline
\end{tabular}

Tabla III. Calibre mayor de las venas superficiales de la fosa cubital, derecha e izquierda, en individuos de ambos sexos, del grupo étnico mapuche, con formaciones venosas Tipo II.

\begin{tabular}{|c|c|c|c|c|c|c|}
\hline & \multicolumn{2}{|c|}{ Lado derecho } & \multicolumn{2}{|c|}{ Lado izquierdo } & \multicolumn{2}{|c|}{ Total } \\
\hline Venas & $\mathrm{n}$ & $\%$ & $\mathrm{n}$ & $\%$ & $\mathrm{n}$ & $\%$ \\
\hline VICo & 37 & 78,8 & 29 & 76,3 & 66 & 77,6 \\
\hline $\mathrm{VC}$ & 5 & 10,6 & 2 & 5,3 & 7 & 8,2 \\
\hline VB & 1 & 2,1 & 1 & 2,6 & 2 & 2,4 \\
\hline $\mathrm{VIB}=\mathrm{VIC}=\mathrm{VICo}$ & 4 & 8,5 & 6 & 15,8 & 10 & 11,8 \\
\hline Total & 47 & 100,0 & 38 & 100,0 & 116 & 100,0 \\
\hline
\end{tabular}

Tabla IV. Frecuencia de la vena intermedia del antebrazo en las formaciones venosas Tipos I, II y III, en individuos de ambos sexos, del grupo étnico mapuche.

\begin{tabular}{|c|c|c|c|c|c|c|c|c|c|c|c|c|}
\hline \multirow[b]{3}{*}{ Grupo I } & \multicolumn{4}{|c|}{ Lado derecho } & \multicolumn{4}{|c|}{ Lado izquierdo } & \multicolumn{4}{|c|}{ Total } \\
\hline & \multicolumn{2}{|c|}{ Mujeres } & \multicolumn{2}{|c|}{ Hombres } & \multicolumn{2}{|c|}{ Mujeres } & \multicolumn{2}{|c|}{ Hombres } & \multicolumn{2}{|c|}{ Mujeres } & \multicolumn{2}{|c|}{ Hombres } \\
\hline & $\mathrm{n}$ & $\%$ & $\mathrm{n}$ & $\%$ & $\mathrm{n}$ & $\%$ & $\mathrm{n}$ & $\%$ & $\mathrm{n}$ & $\%$ & $\mathrm{n}$ & $\%$ \\
\hline Presente & 20 & 43,5 & 12 & 70,6 & 21 & 51,2 & 10 & 83,3 & 41 & 47,1 & 22 & 75,9 \\
\hline Ausente & 26 & 56,5 & 5 & 29,4 & 20 & 48,8 & 2 & 16,7 & 46 & 52,9 & 7 & 24,1 \\
\hline \multicolumn{13}{|l|}{ Grupo II } \\
\hline Presente & 19 & 48,7 & 5 & 62,5 & 17 & 53,1 & 2 & 33,3 & 36 & 50,7 & 7 & 50,0 \\
\hline Ausente & 20 & 51,3 & 3 & 37,5 & 15 & 46,9 & 4 & 66,7 & 35 & 49,3 & 7 & 50,0 \\
\hline \multicolumn{13}{|l|}{ Grupo III } \\
\hline Presente & 16 & 64,0 & 2 & 50,0 & 20 & 58,8 & 7 & 77,8 & 36 & 61,0 & 9 & 69,2 \\
\hline Ausente & 9 & 36,0 & 2 & 50,0 & 14 & 41,2 & 2 & 22,2 & 23 & 39,0 & 4 & 30,8 \\
\hline
\end{tabular}

Tabla V. Drenaje de la vena intermedia del antebrazo en las formaciones venosas Tipos I, II y III de la fosa cubital, en individuos de ambos sexos, del grupo étnico mapuche.

\begin{tabular}{|c|c|c|c|c|c|c|c|c|c|c|c|c|}
\hline \multirow{3}{*}{$\begin{array}{l}\text { Drenaje VIA } \\
\text { Grupo I }\end{array}$} & \multicolumn{4}{|c|}{ Lado derecho } & \multicolumn{4}{|c|}{ Lado izquierdo } & \multicolumn{4}{|c|}{ Total } \\
\hline & \multicolumn{2}{|c|}{ Mujeres } & \multicolumn{2}{|c|}{ Hombres } & \multicolumn{2}{|c|}{ Mujeres } & \multicolumn{2}{|c|}{ Hombres } & \multicolumn{2}{|c|}{ Mujeres } & \multicolumn{2}{|c|}{ Hombres } \\
\hline & $\mathrm{n}$ & $\%$ & $\mathrm{n}$ & $\%$ & $\mathrm{n}$ & $\%$ & $\mathrm{n}$ & $\%$ & $\mathrm{n}$ & $\%$ & $\mathrm{n}$ & $\%$ \\
\hline VB & 2 & 10,5 & 7 & 77,8 & 10 & 50,0 & 6 & 66,7 & 12 & 30,8 & 13 & 72,2 \\
\hline VIB & 5 & 26,3 & 1 & 11,1 & 4 & 20,0 & 2 & 22,2 & 9 & 23,1 & 3 & 16,7 \\
\hline $\mathrm{VC}$ & 12 & 63,2 & 1 & 11,1 & 6 & 30,0 & 1 & 11,1 & 18 & 46,1 & 2 & 11,1 \\
\hline \multicolumn{13}{|l|}{ Grupo II } \\
\hline VICo & 6 & 31,6 & 3 & 60,0 & 5 & 31,2 & 0 & 0,0 & 11 & 31,4 & 3 & 42,9 \\
\hline $\mathrm{VC}$ & 3 & 15,8 & 2 & 40,0 & 5 & 31,2 & 1 & 50,0 & 8 & 22,9 & 3 & 42,9 \\
\hline VB & 10 & 52,6 & 0 & 0,0 & 6 & 37,6 & 1 & 50,0 & 16 & 45,7 & 1 & 14,2 \\
\hline \multicolumn{13}{|l|}{ Grupo III } \\
\hline $\mathrm{VC}$ & 3 & 18,8 & 0 & 0,0 & 5 & 26,3 & 2 & 33,3 & 8 & 22,9 & 2 & 25,0 \\
\hline VB & 13 & 81,2 & 2 & 100,0 & 14 & 73,7 & 3 & 66,7 & 27 & 77,1 & 6 & 75,0 \\
\hline
\end{tabular}




\section{DISCUSIÓN}

Debido fundamentalmente a la sinonimia de los términos abordados, se hizo necesario la utilización de los términos que aparecen en la VI Nomina Anatomica aprobada por el INAC, el año 1985 en Londres y publicada en Edimburgo en 1989. No se utilizó la Terminología Anatómica actual porque dejó de lado las venas mediana cefálica y mediana basílica (intermedias), elementos fundamentales de la división de la VC del antebrazo a nivel de la fosa cubital.

Sin embargo, se debe clarificar un poco más la utilización de la terminología de las venas superficiales del miembro superior, en especial de las venas del antebrazo y de la fosa cubital.

La primera edición de la Nomina Anatomica, la cual fue revisada por el Comité Internacional de Nomenclatura Anatómica designado durante el V Congreso Internacional realizado en Oxford en 1950 y, posteriormente, durante el VI Congreso Internacional de Anatomía efectuado en París en 1955, donde fue aprobada durante una reunión del Comité Internacional de Nomenclatura el 24 de julio del mismo año. Según puede observarse en esta Nomina Anatomica, sólo 2 venas superficiales del miembro superior son destacadas, la vena cefálica y la vena basílica. En la vena cefálica drenarían la venas toracoacromial y la eventual existencia de la vena cefálica accesoria. Por otro lado, y de acuerdo como aparecen indexados los términos anatómicos, en la vena basílica, los vasos que drenarían en ella serían la vena mediana del codo y además, las eventuales venas mediana del antebrazo, mediana basílica y mediana cefálica.

Por otra parte, en la última edición de la VI Nomina Anatomica, revisada y autorizada por el Comité Internacional de Nomeclatura Anatómica (1985) destaca un mayor número de venas superficiales del miembro superior: las venas cefálica (en ella drenarían la vena toracoacromial y la eventual vena cefálica accesoria), basílica, mediana del codo, mediana del antebrazo, mediana cefálica y mediana basílica, entre otras. Como se puede observar, coloca en una misma posición a todas las venas medianas. Creemos que no es posible la coexistencia simultánea de todas ellas; así, si existe la vena cefálica accesoria, ésta se unirá a la vena mediana cefálica una división de la vena cefálica del antebrazo para seguir como vena cefálica en el brazo; pero si no existe la vena cefálica accesoria, la división de la vena cefálica del antebrazo dará la vena mediana del codo que se unirá a la vena basílica para seguir como basílica en el brazo, continuando la vena cefálica del antebrazo como vena cefálica en el brazo.
Finalmente, con respecto a los términos usados para las venas superficiales del miembro superior, la actual Terminologia Anatomica, que es una creación conjunta de la Comisión Federativa de la Terminología Anatómica (FCAT) y de 56 asociaciones que son Miembros de la Federación Internacional de Anatomistas (FIAA), no pudo establecer una relación de trabajo entre el Comité Internacional de Nomenclatura Anatomica (IANC) y la FCAT, y por tanto, las modificaciones efectuadas a la actual terminología son muy pocas. Sin embargo, con respecto al tema de las venas superficiales del miembro superior, sí hay un cambio sustantivo. Así, menciona la existencia de las venas cefálica (drena la vena toracoacromial y la eventual vena cefálica accesoria), basílica, mediana del codo, mediana del antebrazo, cefálica del antebrazo y basílica del antebrazo, dejado nuevamente fuera a las venas mediana basílica y mediana cefálica.

Como se ha establecido en este trabajo, la existencia de la vena cefálica accesoria, determina que las ramas de división de la vena cefálica del antebrazo, y por tanto se les ha denominado venas intermedias (medianas) basílica y cefálica (Tipo I), pero si no existe la vena cefálica accesoria, la división medial de la vena cefálica del antebrazo se denominaría vena intermedia (mediana) del codo.

Finalmente, para poder clarificar por qué se utilizó el término intermedio en vez de mediano (ambos términos considerados en la Nomina y Terminología Anatomica) la Real Academia Española de la Lengua (RAE) considera como mediano (Del lat. medianus, del medio) como de calidad intermedia o también "en un triángulo, la recta trazada desde un vértice al punto medio del lado opuesto" y por otra parte, el término intermedio (Del lat. intermedi us), la RAE lo define como "que está entre los extremos de lugar, tamaño, etc. El término mediano, sea plano o línea, es más utilizado en Anatomía para indicar que se encuentra equidistante entre dos partes (medial y lateral). Si es aplicado a un plano sagital, sería exactamente el que divide, de anterior a posterior, un segmento en dos partes iguales o aparentemente iguales, al igual que lo que ocurre con la recta trazada en el triángulo. Sin embargo, esto no ocurre con el plano intermedio ya el término sólo indica que la estructura se encuentra entre los extremos de un segmento, así sería más correcto, avalado por el trayecto de las venas en el antebrazo, particularmente de la vena cefálica del antebrazo, que asciende desde el tercio distal al proximal del antebrazo en un trayecto oblicuo de lateral a medial. 
Las formaciones venosas de la fosa cubital en los individuos del grupo étnico mapuche, tienen numerosas variaciones al igual que en otro grupos raciales y étnicos. Sin embargo, estas formaciones pueden ser agrupadas, notándose una tendencia hacia la formación de una $\mathrm{M}$ o de una N, dependiendo de la existencia de la VCA.

Nuestras observaciones mostraron que en el 38,7\% las venas a nivel de la fosa cubital formaban una "M", constituida por la VB (medialmente), VCA (lateralmente) y VC (localizada entre ambas); las dos ramas terminales de esta última (VIB y VIC) se unían a la VB y VC, respectivamente. Estas observaciones son similares a las realizadas por Poirier \& Charpy (1902); Bardeleben (1902); Bertelli (1902); Duval (1902); Theile (1902); Berry \& Newton (1908); Chiarugi (1912); Okamoto (1922); Rouvière (1924); Salvi et al. (1932); Skewes (1944) Warwick \& Williams (1979); Woodburne (1984); del Sol et al. (1988) y del Sol \& Olave (1991). Por otra parte, la "M" clásica formada por la división de la VIA, descrita por Testut (1894); Winslow (1902); Paturet (1951) en el 60\% de los casos ; Singh et al. (1982) en el 40\% en nigerianos; Sappey (1876); Tandler (1929); Berry \& Newton en el $16 \%$ en británicos y Charles (1932) en el $19,7 \%$ en americanos blancos y negros, son resultados que nos parecen elevados. Esta "M" clásica sólo representó el 1\% de nuestra muestra, resultado igual al observado por Okamoto en japoneses y muy similar a los relatado por Skewes $(1,7 \%)$ en chilenos de ascendencia hispánica. Una presencia mayor de esta formación, fue relatada por Halim \& Abdi (1974) en hindúes (6,5\%).

Analizando los diferentes conceptos que se tienen de las distintas formaciones venosas en la fosa cubital, referente a la "M" clásica de Winslow, concordamos con Salvi, Chiarugi y del Sol et al. (1988 y 1990) en el sentido que algunos autores consideraban, y aún consideran, erróneamente la "Y" formada por la bifurcación de la VIA en VIC y VIB, lo que creemos es una confusión, ya que la VIA debiese corresponder a la VC y la denominada $\mathrm{VC}$, a la VCA.

Por otra parte, discordamos con la afirmación de Salvi, quien señaló que la VIB corresponde a la VICo y que la VIC corresponde a la VC . Para nosotros esto sólo ocurre en caso de ausencia de la VCA determinado una formación Tipo II. Sin embargo, lo más frecuente, como lo demuestran los resultados $(38,7 \%)$, es la presencia de la VCA y división de la VC, determinando el Tipo I de formaciones venosas de la fosa cubital. De la misma manera, estamos en desacuerdo con Moore (1982), que presenta la M clásica, cuya VIA se divide en dos ramas, una sin nombre y la otra la VICo. Esta última, de acuerdo a la figura presentada, equivale a la VIB, en tanto al VIA, corresponde a la VC y la VC corresponde a la VCA.

De acuerdo a lo anterior y analizando nuestros resultados obtenidos en el Tipo I (38,7\%), éstos son bastante similares a los observados por Charles $(27,5 \%)$ en americanos blancos y negros y por del Sol et al. (1988), en el $30 \%$ de recién nacidos brasileños, por Paturet (30\%) y por del Sol et al. (1990), en el 36,5\% de los jóvenes chilenos. Esta formación venosa se presentó con mayor frecuencia en el miembro superior derecho, independiente del sexo, tal vez por la presencia de individuos diestros. Sin embargo, la VIB, considerando ambos lados y sexos, no resultó ser la de mayor calibre, como lo señalan Bardeleben; Bertelli, Duval y Thiele, pero se debe tener presente que para dichos autores, la VIB es un brazo de la VIA y, en nuestros casos, es una rama de división de la VC. La VIA, presente en el 54,3\% de los casos de Tipo I se encontraba en mayor porcentaje en individuos de sexo masculino debido, tal vez, a la mayor masa muscular y a la actividad física realizada. Esta VIA drenaba principalmente en la VB.

El Tipo II, donde la VC origina la VICo, fue observada en un $28,3 \%$ considerando ambas fosas cubitales y ambos sexos, determinando el Tipo II. Estos resultados obtenidos en individuos del grupo étnico mapuche, son similares a los observados en recién nacidos brasileños (30\%) por del Sol et al. (1988) y en jóvenes chilenos (28,75\%) por del Sol et al. (1990). Sin embargo, son bastante diferentes a los elevados porcentajes encontrados por Berry \& Newton en el $83 \%$ de los británicos y por Charles, en el $70 \%$ de los americanos blancos y negros y, a los bajos porcentajes encontrados por Paturet (8\%) y por Singh et al., en el 11\% de los nigerianos.

Considerando juntos los Tipos I y II y comparándolos con los datos de otros investigadores, tenemos: $87 \%$ en chilenos de ascendencia hispánica, $85,5 \%$ en japoneses; $67,5 \%$ en hindúes; $67 \%$ en mapuches; $65 \%$ en jóvenes chilenos; y $60 \%$ en recién nacidos brasileños. Valores muy diferentes son reportados por Sohier et al. (1964, 1969) quienes determinaron disposiciones clásicas tipo M o aparentes en sólo el 38,1\% de los casos en las venas superficiales del pliegue del codo en africanos de África Occidental.

En el Tipo II, la vena de mayor calibre fue la VICo, considerando ambo sexos y ambos lados, coincidiendo con las informaciones de del Sol et al. (1990) en jóvenes chilenos y del Sol \& Olave, pero diferente a los resultados obtenidos por del Sol et al. (1988) en recién nacidos brasileños. Por otra parte, autores como Chiarugi han se- 
ñalado que la VB es más voluminosa que la VC y que la VICo es una gran rama de la VC. Sin embargo, debe tenerse presente que lo señalado por Chiarugi puede ser considerado tanto para la formación Tipo I, como para la Tipo II, ya que este autor no efectúa esta clasificación. Similares observaciones fueron realizadas por Orts Llorca (1952) en relación a que la VICo se torna voluminosa y después la $\mathrm{VC}$ prosigue en el brazo como una pequeña vena. Aún no teniendo referencia en la literatura sobre el drenaje de la VIA en el Tipo II, hemos observado que, al igual que en el Tipo I, la VIA drena en la VB.

En el Tipo III, en que no existe comunicación entre la VC y VB a nivel de la fosa cubital, se presentó en el $24 \%$ de los individuos mapuche, considerando ambos lados y ambos sexos, en tanto Berry \& Newton, encontraron este tipo de formación venosa sólo en el 1\% de los británicos, Singh et al., en el 10\% de los nigerianos, del Sol et al. (1988) en el 25\% brasileños, del Sol et al. (1990) en el $17,25 \%$ de los jóvenes chilenos y Halim \& Habdi en el 12,5 en hindúes. Estos últimos autores, subdividen el Tipo III de nuestras observaciones, en el Tipo III-A (6\%) y en el Tipo III-B o "M” clásica (6,5\%).

El Tipo III se presentó en los individuos mapuche con mayor frecuencia en la fosa cubital izquierda, en ambos sexos, siendo esta diferencia más notoria en el sexo masculino. Estos resultados contrastan con los obtenidos en los Tipos I y II, donde las respectivas formaciones venosas fueron más frecuentes en el lado derecho. Con respecto al drenaje de la VIA $(65,2 \% \%$ de presencia), ésta drenaba generalmente en la VB, concordando con los resultados de del Sol et al. (1990) en jóvenes chilenos y del Sol \& Olave, pero diferente a lo observado en brasileños en los cuales la VIA podía estar ausente o drenar en la VB o VC. Para por Sohier et al. (1964, 1969), la disposición de tipo embrionario, definida por ellos como Tipo II y Tipo III, ocurre en la mayoría de los casos $(62 \%)$ en las venas superficiales del pliegue del codo, en africanos de África Occidental. Sin embargo, hay que señalar que estas disposiciones no corresponden a la división planteada por nosotros, ya que los Tipos II y III de Sohier et al. $(1964,1969)$ serían semejantes al Tipo IV de nuestro estudio, pero con diferencias ya que para el Tipo II señalan que la VB es predominante y recoge casi la totalidad de la sangre del antebrazo, drenando en ella una gran VIA y siguiendo por el brazo una pequeñísima VC. Por otra parte, el Tipo III de Sohier et al. $(1964,1969)$ correspondería al Tipo IV de nuestro estudio.

El Tipo IV presente en sólo el 4,3\%, es bastante menor a lo observado por Halim \& Abdi (19,5\% en individuos hindúes) y por Singh et al. (10\% en nigerianos). En cambio, los resultados obtenidos por Charles $(1,6 \%$ en individuos americanos blancos y negros) se aproximan a los nuestros. Cabe destacar, la ausencia de este tipo de formación venosa en los individuos japoneses (Okamoto). Por otra parte, la VIA presente en el $61,5 \%$, drena en el $100 \%$ de los casos en la VC, lo que resulta lógico desde el punto de vista anatómico ya que la VC se dirige hacia medial y hacia el brazo, alcanzando a la VB antes de que ésta perfore la fascia y transcurra profunda por el resto de su recorrido.

En el Tipo V, donde están incluidas el resto de las formaciones venosas, hemos incluido a la "M" clásica descrita Por Winslow y que para Chiarugi no existe. Esta formación representó apenas el $1 \%$ de nuestro estudio, por tanto debe ser considerada como una variación anatómica. No obstante lo anterior, la "M" clásica para Testut y otros autores es una formación común, siendo muy frecuente para Tandler y Orts Llorca. Concluimos que la "M" clásica en individuos mapuche, es extremadamente infrecuente, concordando así con las observaciones realizadas por Poirier \& Charpy, Salvi, Chiarugi, Charles, Halim \& Abdi y del Sol et al. (1988 y 1990) y a la vez discordando a lo indicado por otros autores como Sappey, Winslow, Testut, Paturet y Singh et al., para quienes la existencia de esta formación es la más común.

Es importante conocer las formaciones venosas de la fosa cubital ya que éstas mantienen estrechas relaciones con otras estructuras anatómicas de la región. Normalmente, estas venas están en relación con la arteria braquial que, a nivel de la fosa cubital, se divide en las arterias ulnar y radial. Por tal motivo y, a pesar que recomendamos la utilización de la VB, por su mayor calibre en el Tipo I, debemos recordar que, profunda a ella, se encuentra la arteria braquial y el nervio mediano, estructuras separadas únicamente por la expansión bicipital. Asímismo, ramos anteriores del nervio cutáneo medial del antebrazo cruzan, generalmente la cara anterior de la VIB o de la VICo y que, cuando son alcanzados, provocan un dolor agudo en dicha región. Por lo anterior, recomendamos la utilización de la VIC o VC cuando estas venas presentan un calibre semejante a la VIB o VICo, porque los riesgos de punción arterial o nervioso son improbables, ya que la arteria braquial y el nervio mediano se encuentran alejados de estas venas, como también, el nervio cutáneo lateral del antebrazo, ramo terminal del nervio musculocutáneo, presenta ramos en general retrovenosos.

Finalmente, el abordaje de cualquier vena de la fosa cubital, para la extracción de sangre, administración medicamentosa, introducción de catéteres cardiacos, denudación venosa, entre otras, debe ser realizado con mucha precaución, teniendo presentes las observaciones antes señaladas y considerando además, las numerosas variaciones anatómicas presentes en la región cubital (del Sol et al., 2000). 
DEL SOL, M.; LAGOS, M.M.\& TORRES, B.E. Venous formations in the cubital fossa of mapuche. Bioscopy study Int.J.Morphol., 25(4):885-894, 2007.

SUMMARY: The superficial veins of the cubital fossa constitute one of the most important sites for vein puncture. The availability of those veins present numerous variations, and the availability of these has not been studied in the Mapuche ethnic group. In view of the above, and considering this group as the greatest ethnic conglomerate in South America the study took place. The superficial veins of the cubital fossa were analized in 300 superior members (150 right and 150 left) in subjects of both sexes (30 men and 120 women) Chileans of the Mapuche ethnic group between 15 and 84 years of age. The study was realized in rural clinics near the city of Temuco. Based on the classification of del Sol et al. (1988) for the vein formation of the cubital fossa, the following results were obtained: type I (38.7\%), the cephalic vein (VC) is divided in the intermediate basilic vein (VIB) and intermediate cephalic vein (VIC), joining thebasilic vein (VB)and accessory cephalic vein (VCA) respectively. The VIB was of a major caliber and the intermediate vein of the forearm (VIA) generally drained in the VB, Type II $(28,3 \%)$, the VC originates the intermediate vein of the elbow (VICo), that joins the VB. VCA does not exist, th VICo being of a mayor caliber and the VIA drains in to VB, Type III (24\%) no communication exists between the VB and VC at the level of the cubital fossa, the VIA drains into VB, Type IV (4,3\%), the VC drains in the VB and VIA drins in the VC; Type V other dispositions where the classic M is included (1\%) which results in the division of the VIA. The use of the VIC and VC is recommended when they have a similar caliber to that of the VIB of VICo. Because the puncture risks of other important anatomic structures, such as anterior branches of the medial cutaneous nerve of the forearm or brachial artery are minimal.

KEY WORDS: Anatomy; Cubital fossa veins; Sites of vein puncture.

\section{REFERENCIAS BIBLIOGRÁFICAS}

Bardeleben apud Poirier, P. \& Charpy, A. 1902, op. cit.

Berry, R. J. A. \& Newton, H. A. S. A study of the superficial veins of the superior extremity in 300 living subjects. Anat. Anz., 33(5):591-601, 1908.

Bertelli apud Poirier, P. \& Charpy, A. 1902, op. cit.

Cagnon, R. Superficial arteries of the cubital fossa with reference to accidental intra-arterial injections. Can. J. Surg., 9:57-65, 1966.

Charles, C. M. On the arrangement of the superficial veins of the cubital fossa in American white and American negro males. Anat. Rec., 54:9-14, 1932.

Chiarugi, G. Istituzioni di Anatomia dell'Uomo. Milano, Societa Editrice Libraria, 1912. V.2.

Comité International de la Nomenclature Anatomique. Nomina Anatomica. Ballantyne, Londres, 1985. (Imprimé à titre de publication privée).

Del Sol, M. Indice cefálico en un grupo de individuos mapuches de la IX Región de Chile. Int. J. Morphol., 23(3):241-6, 2005.

Del Sol, M. Índices faciales en individuos mapuche. Int. $J$. Morphol., 24(4):587-90, 2006.
Del Sol, M.; De Angelis, M. A. \& Bolini, P. A. D. Formações venosas na fossa cubital da criança. Pediatria Moderna, 23 (4):225-31, 1988.

Del Sol, M. \& Hunter, K. Evaluación postural de individuos mapuche de la zona costera de la IX Región de Chile. Int. J. Morphol., 22(4):339-42, 2004.

Del Sol, M.; Wuster, A. \& Fritz, I. Tipos de formaciones venosas en la fosa cubital en jóvenes chilenos. Rev. Chil. Tecnol. Méd., 13(2): 646-9, 1990.

Del Sol, M. \& Olave, E. Venas de la fosa cubital en el hombre. Sitios de punción. Rev. Chil. Cs Med. Biol., I(1):49$53,1991$.

Del Sol, M.; Olave, E.; Hettich, M. E. \& Bustos, K. Arteria ulnar superficial: importancia anátomo-clínica. Rev. Chil. Anat., 18 (1): 2000.

Duval, M. apud Poirier, P. \& Charpy, A. 1902, op. cit.

Federative Committee Anatomical Terminology (FCAT). Terminologia Anatomica. International Anatomical Terminology. Thieme, New York, 2000.

Guevara, T. La complexión física del araucano. In: Historia de Chile. Chile Prehispánico. Balcells, Santiago de Chile, 1927. V 2. pp. 321-9. 
Halim, A. \& Abdi, S. H. M. Superficial venous patterns in the cubital region of Indians. Anat. Rec., 178(3):631-6, 1974.

Henckel, C. Antropología Física de los mapuches. Rev. Universitaria, 43(22):13-22, 1958.

International Anatomical Nomenclature Committee. Nomina Anatomica. $6^{\text {th }}$. ed. Edinburgh, Churchill Livingstone, 1989.

Last, R. J. Superficial veins of the forearms. The surgical anatomy in relation to intravenous injection. Brit. Dental J. 125(11):491-4, 1968.

Moore, K. L. Anatomía. Buenos Aires, Panamericana, 1982.

Okamoto, K. A study of the superficial veins in the superior extremity of live Japanese. Anat. Rec., 23:323-33, 1922.

Orts Llorca, F. Anatomía humana. Barcelona, Científica Médica, 1952.V. 3.

Paturet, G. Traité d'anatomie humaine. Paris, Masson, 1951. V. 2.

Poirier, P. \& Charpy, A. Traité d'anatomie humaine. 2. ed.Paris, Masson, 1902. V. 3.

Real Academia Española. 22a . ed. Diccionario de la Lengua Española, Espasa-Calpe, Madrid, 2001.

Rouvière, H. Anatomie humaine. Paris, Masson, 1924. V.2.

Salvi, G. Angiología in Balli, R.; Bertelli, D. et al. Trattato di anatomia umana. 2a . ed. Milano, Casa Editrice Dottor Francesco Vallardi, 1932. V.3.

Sappey, $\mathrm{Ph}$. Traité d'anatomie descriptive. $3^{\mathrm{a}}$. ed. Paris, Adrien Delahaye, 1876. V. 2.

Singh, S. P. ; Ekandem, G. J. \& Bose, E. S. A study of the superficial veins of the cubital fossa in Nigerian subject. Acta Anat., 114:317-20, 1982.

Skewes, E. Estudio de las venas superficiales del antebrazo en los chilenos. Bol. Soc. Biol., 19:75-81, 1944.

Sohier, H. M. L.; Fustec, R. \& Laffont, J. Veines superficielles du pli du coude chez l'Africain d'Afrique occidental. Bul. Soc. Méd. Afrique Noire, 7(1):107-11, 1962.
Sohier, H. M. L.; Fustec, R.; Laffont, J. \& Le Guyader, A. Veines superficielles du pli du coude chez l'Africain d'Afrique occidental. Bull. Assoc. Anatomistes, 120:1230-5, 1964.

Tandler, J. Tratado de anatomía sistemática. Barcelona, Salvat, 1929.

Testut, L. Trattato di anatomía umana. Torino, Unione tipografico- Editrice, 1894.V.2.

Thiele apud Poirier, P. \& Charpy, A. 1902, op.cit.

Warwick, R. \& Williams, P. L. Gray-Anatomia. 35 ${ }^{\mathrm{a}}$. ed. Rio de Janeiro, Guanabara Koogan, 1979. V.1.

Winslow apud Poirier, P. \& Charpy, A. 1902, op.cit.

Woodburne, R.T. Anatomía humana. 6a . ed. Rio de Janeiro, Guanabara Koogan, 1984.

Dirección para correspondencia:

Prof. Dr. Mariano del Sol

Facultad de Medicina

Universidad de La Frontera

Casilla 54-D

Temuco

CHILE

Recibido: 10-08-2007

Aceptado: 20-10-2007 